\title{
La metafísica de la consolación de Moses Mendelssohn
}

\author{
The consoling metaphysics \\ of Moses Mendelssohn
}

\author{
Pablo Alejandro ARIAS PÉREZ \\ Universidad Complutense de Madrid
}

Recibido: 21/11/2012

Aceptado: 07/05/2014

\section{Resumen}

Moses Mendelssohn, conocido por lo general por su contribución a la emancipación y modernización de la sociedad judía, fue también uno de los más importantes filósofos ilustrados alemanes. Sin embargo, su obra no ha merecido mayor atención durante bastante tiempo en parte debido a que sus argumentos de la inmortalidad del alma, Dios, así como su concepto de la providencia se quedaron al poco tiempo obsoletos con la aparición de la filosofía crítica kantiana. En este sentido, se le consideraba como un pensador más de la tradición clásica racionalista alemana en la misma línea que Wolff o Baumgarten o llegaba incluso a ser tachado de ser filósofo popular. No obstante, su obra merece ser considerada bajo una nueva perspectiva. Mendelssohn no solamente se limitó a concebir los argumentos tradicionales bajo una forma nueva, sino que intuyó este nuevo tiempo, tratando de salvaguardar las ideas metafísicas más importantes a través de demostraciones que recurrían al argumento práctico, algo que le convierte en un precursor de la filosofía crítica.

Palabras clave: ilustración, racionalismo, crítica, especulación, entendimiento sano. 


\begin{abstract}
Moses Mendelssohn, generally known for his contribution for the emancipation of Jews was besides one of the most important German enlighteners. His oeuvre has been commonly ignored for a long time due to the fact that his demonstrations of the immortality of the soul, God and his concept of providence were soon outdated with the birth of Kants critical philosophy. While most of the scholars considered him as a philosopher of the German old rationalist school in the line of Wolff and Baumgarten, or even branded him as a popular philospher, his work should be reexamined in a new light. For he didn't just remained faithful to the old classic metaphysical demonstrations, providing them with a new form, but rather he also guessed at this new time coming. With this in mind he tried to safeguard the main metaphysical ideas resorting to a practical approach. This makes him a forerunner of the critical philosophy.
\end{abstract}

Keywords: enlightenment, rationalism, critique, speculation, common sense.

\title{
1. Introducción
}

En este artículo se muestra una idea general de la problemática de la metafísica de Mosés Mendelssohn. Se trata de una exposición detallada de los principios metafísicos, Dios, Inmortalidad del alma y providencia, los principios de la religión natural o religión de la razón, haciendo especial hincapié en las demostraciones y su evolución a lo largo de su trayectoria. Por un lado, la defensa de estos principios estuvo acompañada en todo momento por un escepticismo cada vez mayor con respecto a la argumentación demostrativa que se materializó finalmente en un cuestionamiento de la capacidad misma de la razón. Por otro lado, Mendelssohn rechazó en todo momento la idea de revelación exclusiva de principios metafísicos tal y como hiciera gran parte del movimiento ilustrado. Por lo tanto, estos dos factores, el creciente escepticismo y el rechazo de la revelación exclusiva de principios de la religión natural marcaron el desarrollo de la filosofía mendelssohniana desde la aparición de su Abhandlung über die Evidenz en 1763 hasta las Morgenstunden de 1786. La elaboración de una facultad alternativa a la razón, capaz de alcanzar estas verdades universalmente ocupará gran parte del pensamiento mendelssohniano y culminará en su concepto de entendimiento sano, tal y como es formulado en 1786. El ímpetu con el que Mendelssohn se afana en salvaguardar estas verdades no obedece a una 
posición dogmática sino que está dictada por su convencimiento de que estas verdades son imprescindibles para la felicidad humana. En el presente artículo se pondrá de manifiesto la confluencia de estrategias de Mendelssohn a la hora de avanzar hacia un mismo fin: asegurar la felicidad humana.

La confluencia en favor de la felicidad del hombre pone de manifiesto lo que en términos kantianos podría ser vista como una primacía de la razón práctica sobre la teórica, que hace de Mendelssohn un auténtico pensador moderno.

\section{Influencias, contexto y primeros escritos}

La metafísica mendelssohniana se basa en la idea de una capacidad común a todos los hombres para la comprensión de los principios de la religión natural. La demostrabilidad racional de la religión natural es algo que Mendelssohn toma por un lado de Leibniz y Wolff pero igualmente de otros filósofos judíos anteriores. Especialmente Maimónides e Ibn Ezra son dos de los más importantes filósofos que intentaron probar la idea de Dios como una verdad universal y de razón. ${ }^{1}$ Por otro lado, la filosofía de Mendelssohn puede ser ubicada dentro el pensamiento deísta, especialmente en lo que respecta a la relevancia de la religión de la razón y su relación con la felicidad humana. De igual manera ha de ser destacada la notoria influencia del procedimiento sistemático de Wolff en la demostración de la religión natural. ${ }^{2}$

En la época en que Mendelssohn comienza su carrera se empieza a producir una convulsión del modo teórico en la demostración de los enunciados de la religión natural, fruto de un escepticismo creciente. La filosofía de la religión mendelssohniana tiene por tanto la particularidad de haber nacido en un terreno marcado profundamente por la racionalidad del procedimiento teórico aunque es consciente en todo momento del escepticismo incipiente. La convergencia de estos dos hechos se evidencia en el ensayo de Mendelssohn de 1763 Abhandlung über die Evidenz in metaphysischen Wissenschaften (Tratado sobre la evidencia en las ciencias metafisicas).

El Abhandlung über die Evidenz... es concebido como ensayo a modo de pregunta lanzada por la Real Academia de las Ciencias de Berlin (Königliche Akademie der Wissenschaften in Berlin) acerca de sí las verdades metafísicas

${ }^{1}$ Cfr. Jospe R., "Moses Mendelssohn, A medieval Modernist", en varios (Eds.), Jewish Philosophy. Foundations and Extensions. Vol. 2: On Philosophers and their Thought, Lanham, University Press of, 2008, pp. S.205-231, aqui p.210.

${ }^{2}$ Cassirer E., Die Philosophie Moses Mendelssohns, en Gesammelte Werke, Vol. 17, Hamburgo: Meiner Verlag, 2009, pp.115-138, aquí p.116 y siguientes. 
en general y en particular los principios primeros de la teología natural y de la moral pueden tener la misma evidencia que las verdades matemáticas, y en caso contrario, cual es la naturaleza de su certeza y que grado puede alcanzar así como si este es adecuado para la convicción". ${ }^{3}$ La academia de las ciencias, institución fundada por Federico I, concedía este premio anual a un ensayo entregado anónimamente cuyo autor era revelado una vez conocido el fallo. La ironía de la historia hizo que el Kant pre-crítico alcanzará el segundo puesto después de Mendelssohn.

El grado de certeza que Mendelssohn reconoce a la ciencia matemática se debe a que las matemáticas son una ciencia conceptual puramente cuantitativa que no maneja cualidades de la realidad. ${ }^{4}$ En este sentido, ya al principio de su ensayo reconoce que las ciencias metafísicas no pueden alcanzar el mismo grado de evidencia que las matemáticas:

"Incluso aquellos que consideran los conceptos metafísicos como convicciones irrefutables, han de reconocer finalmente que aún no poseen la evidencia de las pruebas matemáticas, ya que de otra manera no se encontraría en ellas tantas variadas contradicciones". ${ }^{5}$

La evidencia de una verdad se compone de comprensibilidad y certeza. Es cierto que por un lado la certeza de las verdades metafísica está fuera de toda duda, no así, en cambio, su comprensibilidad. Las verdades metafísicas no están dotadas de fuerza de convicción y comprensibilidad. ${ }^{6}$ Ello se debe se deben a la importancia vital de estas sentencias metafísicas, ${ }^{7}$ de manera que

${ }^{3}$ El texto como tal decía: "ob die metaphysischen Wahrheiten im allgemeinen, und im Besonderen die ersten Prinzipien der natürlichen Theologie und der Moral der selben Evidenz fähig sind wie die mathematischen Wahrheiten, und, falls sie deren nicht fähig sind, welches die Natur ihrer Gewißheit ist, bis zu welchem Grad sie gelangen kann, und ob dieser Grad zur Überzeugung genügt“" (M. Brocke, D. Krochmalnik, E.J.Engel y A. Altmann (eds.), Gesammelte Schriften. Jubiliäumausgabe, Stuttgart-Bad Cannstatt, Fromann Holzboog, 1972 en adelante (de aquí en adelante JubA), aquí L.Strauss/Bamberger, "Einleitung" JubA 2, p.XLV y siguientes.

${ }^{4}$ Mendelssohn M., Abhandlung über die Evidenz in den metaphysischen Wissenschaften (1763) en JubA 2 S.281f., pp. 288-289.; cfr. también L.Strauss/Bamberger, «Einleitung», en JubA 2, p. XLVII.

5 "Selbst diejenigen, welche die metaphysische[n] Begriffe für Überzeugungen und unwiderlegbar halten, müssen doch endlich gestehen, daß man ihnen noch bisher die Evidenz der mathematischen Beweise nicht gegeben hat, sonst hätten sie unmöglich einen so vielfältigen Widerspruch finden können. (Mendelssohn, M. Abhandlung über die Evidenz in den metaphysischen Wissenschaften (1763) en JubA 2, p. 271).

${ }^{6}$ Ibid., p. 272.

${ }^{7}$ Ibid., p. 295. 
cualquiera puede formarse un pre-juicio ya que cada uno tiene el poder de ejercer la "magistratura" (Richteramt). ${ }^{8}$

La libertad de análisis es una condición innegociable en la república de la sabiduría mundana, ${ }^{9}$ a pesar de que de ello se derive un verdadero peligro de error en relación a las verdades metafísicas. Por esta razón, para Mendelssohn es indispensable que estén aseguradas por la razón.

A pesar de la afinidad con la metafísica escolar ${ }^{10} \mathrm{y}$ del empeño demostrativo tan propio de esta, se puede constatar, pues, como ya al principio de su trayectoria filosófica Mendelssohn deja entrever cierta insatisfacción con su método científico sobre todo en lo referente a la suficiencia de su método universal. Esta insatisfacción inicial desembocará finalmente en un giro hacia el entendimiento sano como fuente cognitiva. Sin embargo, en el medio se sucede una serie de demostraciones de los principios metafísicos básicos, Dios, inmortalidad del alma y providencia, en las que Mendelssohn irá desarrollando y mejorando las pruebas existentes haciendo uso en numerosas ocasiones de demostraciones en clave práctica e intuitiva tal y como se expondrá a continuación.

\section{Argumentos de la existencia de Dios}

Los argumentos de la existencia de Dios de Mendelssohn están marcados a modo general por una transición desde una deducción meramente racionalista a un método más intuitivo. Este hecho se aprecia tanto en el argumento cosmológico como en el ontológico.

Los primeros argumentos son presentados en la Abhandlung über die Evidenz. En este ensayo Mendelssohn indaga acerca de cómo puede ser probada la existencia partiendo de la posibilidad racional, es decir, si es posible una

\footnotetext{
${ }^{8}$ Ibid., p. 296.

${ }^{9}$ El término "Weltweisheit" se remonta a Wolff. En el párrafo 1 de la Lógica se encuentra la siguiente definición: "La sabiduría del mundo es la ciencia de todas las cosas posibles, del cómo y por qué son posibles" ("die Weltweisheit ist eine Wissenschaft aller möglichen Dinge, wie und warum sie möglich sind" (Wolff, C., Vernünftige Gedanken (I) Logik (1713), en Gesammelte Werke, J.École, H.W. Arndt, Ch. A. Corr, J.E. Homann y M. Thomann (eds. ), tres secciones (Abt.), Hildesheim/Nueva York, Olms, 1962 en adelante (de aquí en adelante VW), aquí VW Abt. I Bd. 1, Vorbericht von der Weltweisheit, p. 115).

${ }^{10}$ Bajo el concepto de Schulmetaphysik se entiende la filosofía predominante en el barroco alemán que se enseñaba en escuelas, universidades y liceos y que estaba caracterizada por una unión estrecha con la teología y la defensa teórica de los principios de esta. Su influencia se mantuvo hasta mediados del siglo XVIII. Consultar la monografía de Max Wundt, Die deutsche Schulmetaphysik des 17. Jahrhunderts, Hildesheim/Zúrich/Nueva York, Georg Olm Verlag, 1992.
} 
deducción lógica de la existencia partiendo de la mera posibilidad. Tanto el argumento ontológico como el cosmológico tienen pues la tarea de justificar este "salto". Además de estos dos argumentos existe el argumento del Cogito, que parte del "yo pienso" para llegar al "Dios existe".

\subsection{Argumento cosmológico}

El argumento cosmológico es concebido en el Ensayo sobre la Evidencia como una alternativa al argumento ontológico. Para ello Mendelssohn se orienta en gran medida en el argumento wolffiano y su enunciado fundamental de la determinabilidad de lo existente: "quicquid existit vel act est, id omnimode determinatum est." $\mathrm{El}$ resto del argumento viene dado por la determinación del individuo deducida de ese mismo enunciado así como del Principium individuationis. ${ }^{12} \mathrm{El}$ argumento propiamente de Mendelssohn parte de la contingencia de las cosas, puesto que estas pueden tanto existir como no hacerlo, al contrario que la existencia del ser perfecto que es algo intrínsecamente necesario a su esencia. Además, este ser perfecto ha de contener en sí mismo la razón de su existencia, puesto que en él, sujeto y predicado (la existencia) son lo mismo. Las cosas contingentes, por su parte, no están determinadas por sí mismas ni a la existencia ni a otros predicados, sino que dependen de otros para sus predicados y determinaciones. En el caso del ser perfecto, las determinaciones son intrínsecas él mismo. Aludiendo al principio de razón suficiente, Mendelssohn deduce que el ser más perfecto ha de ser también la razón de las cosas contingentes y él es la condición indispensable para su existencia y determinación. ${ }^{13}$ De esta manera, la contingencia de las cosas conduce de manera lógica a la necesidad del ser perfecto. ${ }^{14}$

Mendelssohn continúa su argumento cosmológico en la obra de 1786 Morgenstunden oder Vorlesungen über das Daseyn Gottes (Lecciones matinales sobre la existencia de Dios) en la que reformula el argumento de la existencia de Dios bajo tres formas: el argumento de creación, de contingencia y de insuficiencia. En el primer argumento se trata el principio de causalidad y la

\footnotetext{
${ }^{11}$ Wolff C., Philosophia Prima sive Ontologia (1730), en VW Abt. II Vól.3, párrafo 226, pág.187; cfr. Altmann, A. : Mendelssohns Frühschriften zur Metaphysik, Tübingen, MohrSiebeck, 1969, p. 318.

${ }^{12}$ Wolff C., VW Philosophia Prima sive Ontologia (1730), en VW Abt. II Vol.3 párrafo 227 y siguientes. p.188 y siguientes; veáse también Altmann, Mendelssohns Frühschriften zur Metaphysik, p. 317.

${ }^{13}$ Mendelssohn, M., Abhandlung über die Evidenz (1763), en JubA 2, p. 300 y p. 303; cfr. Altmann A., Frühschriften zur Metaphysik, p. 319.

${ }_{14}$ Mendelssohn, M., Abhandlung über die Evidenz (1763), en JubA 2, p. 304.
} 
temporalidad del mundo. La imposibilidad de una línea sin fin se deduce del principio según el cual sin un comienzo "la línea de cosas no puede haber llegado a ser real". ${ }^{15}$ Mendelssohn constata en el capitulo doce del ensayo que este argumento no se sostiene por sí mismo, ${ }^{16}$ volviendo su atención sobre el argumento de contingencia. Para ello se sirve de la forma dada por Leibniz. ${ }^{17}$ En realidad, se trata del mismo argumento del Abhandlung über die Evidenz encaminado a la demostración de un ser perfecto y necesario partiendo de la insuficiencia de las cosas contingentes. Además, Mendelssohn se apoya claramente en Leibniz al trazar la diferenciación entre el entendimiento divino como lugar de las verdades eternas y la voluntad divina como lugar de las verdades contingentes. ${ }^{18}$

A pesar del empeño de Mendelssohn en formular un argumento más sutil en esta última obra, resulta evidente que del antiguo argumento de la contingencia, incluso en su nueva forma, se deduce una causa del mundo pero no un ser absolutamente necesario apoyado sobre sí mismo. Con un resultado similar Mendelssohn intenta revestir de una nueva forma al argumento cosmológico mediante el argumento de la insuficiencia del espíritu subjetivo, según el cual es necesario un entendimiento objetivo e infinito capaz de pensar todas las cosas posibles y reales. ${ }^{19}$

\subsection{Argumento ontológico}

El primer argumento que expone Mendelssohn de manera breve en la Abhandlung über die Evidenz es el argumento ontológico de la existencia, para el que se apoya en Baumgarten. ${ }^{20}$

La demostración comienza por el "no-ser" (Nichtseyn) pasando a las cosas existentes, que están marcadas por su determinación. No obstante, la “existen-

15 “(..) die Reihe der Dinge nicht zu Wirklichkeit” gekommen sein könne (...) " en Mendelssohn, M., Morgenstunden oder Vorlesungen über das Daseyn Gottes en JubA 3.2, p.92; cfr. Altmann, A., "Moses Mendelssohns Proofs for the Existence of God", en Schulte C., y Klein, H.G. (eds.), Mendelssohn Studien 2, 1975, pp. 9-30, aquí p.12 en adelante; Altmann A., Frühschriften zur Metaphysik, p. 318.

${ }_{16}$ Mendelssohn, M. Morgenstunden oder Vorlesungen über das Daseyn Gottes, en JubA 3.2, p. 93.

${ }_{17}$ Altmann A., Proofs for the Existence of God, p. 16.

${ }_{18}$ Mendelssohn, M. Morgenstunden oder Vorlesungen über das Daseyn Gottes, en JubA 3.2, p. 95 en adelante; cfr. tambien Altmann A., Moses Mendelssohns Proofs for the Existence of God, p. 17 y siguientes.

${ }_{19}$ Mendelssohn, M., Morgenstunden oder Vorlesungen über das Daseyn Gottes, en JubA 3.2, p. 144 y siguientes; cfr. tambien Altmann A., Proofs for the Existence of God, pp. 18-19.

${ }^{20}$ Bourel D., Moses Mendelssohn. La naissance du judaïsme moderne, Paris, Gallimard, 2004, p.171. 
cia" (Daseyn) de estas cosas no es algo intrínseco a su esencia, sino pura contingencia y por ello dependiente de algo externo. Esto es simplemente imposible para el ser más prefecto, puesto que si su existencia fuera contingente, se darían contradicciones en su ser. En Dios no puede haber contradicciones, puesto que éstas surgen cuando una cosa tiene carencias y limitaciones, algo incompatible con el concepto de Dios. ${ }^{21}$ No obstante, estas especulaciones, certeras y lógicas, sólo sirven para probar la existencia de un ser perfecto, pero no necesario. ${ }^{22}$

En las Morgenstunden Mendelssohn recurre de nuevo al argumento ontológico. En la formulación del argumento ontológico utilizada aquí se intuye en cierto modo una toma de conciencia de la refutación kantiana en su Crítica de la razón pura. ${ }^{23}$ De acuerdo a esta nueva visión del argumento ontológico, Mendelssohn pretende mostrar que probando la mera posibilidad del concepto de Dios, su realidad queda igualmente demostrada. La premisa subyacente es la identidad entre concepto y cosa. Para la demostración Mendelssohn retoma la suposición según la cual la existencia es tan solo un añadido a la cosa y no puede ser obtenida del concepto mismo, tal y como afirman los oponentes del argumento ontológico. ${ }^{24}$ Precisamente la toma en consideración de las razones de los contrarios al argumento ontológico sirve a Mendelssohn para pasar a un argumento construido sobre el entendimiento sano (gesunder Menschenverstand). La necesidad racional objetiva del concepto de Dios, es decir, el reconocimiento universal de una sentencia de razón entre los entes racionales, se convierte en la prueba decisiva de la existencia de Dios. ${ }^{25}$ Por tanto, el argumento ya no tiene una base especulativa o demostrativa, sino que su evidencia se deduce de una regla del pensamiento, de una intuición natural, con apariencia lógica y necesaria pero que no supera un examen crítico. Con este paso Mendelssohn se introduce de pleno en el reino del entendimiento sano.

${ }^{21}$ Mendelssohn, M., Abhandlung über die Evidenz, en JubA 2, p.300; cfr. Altmann, A. Mendelssohns Frühschriften zur Metaphysik, p. 303.

${ }^{22}$ Cfr. op. cit., p.303.

${ }^{23}$ Altmann A., Moses Mendelssohns Proofs for the Existence of God, pp. 25-26.

${ }^{24}$ Mendelssohn, M., Morgenstunden oder Vorlesungen über das Daseyn Gottes, en JubA 3.2, pp. 149 y siguientes.

${ }_{25}$ Mendelssohn, M., Morgenstunden oder Vorlesungen über das Daseyn Gottes, en JubA 3.2, p. 155. 


\section{Inmortalidad del alma}

En la obra de 1767 Phaedon oder über die Unsterblichkeit der Seele (Fedón o sobre la inmortalidad del alma) Mendelssohn se ocupa detalladamente de la inmortalidad del alma. Gracias a esta importante obra del siglo XVIII Mendelssohn alcanza un papel protagonista en los círculos ilustrados alemanes a la vez que obtiene un enorme éxito tal y como demuestra su denominación a partir de entonces como el "Sócrates alemán". ${ }^{26}$

La intención primaria de Fedón consiste en dotar al argumento de la inmortalidad de una forma más ágil y sencilla. Para este fin Mendelssohn decide redactar la obra como un diálogo. Además, rehúsa presentar un argumento con un fundamento religioso y es por ello que escoge la figura de Sócrates como protagonista del diálogo. ${ }^{27}$

Este tratado contiene tres argumentos. El primero se remonta al Fedón de Platón mientras que el segundo lo hace a la obra de Plotino Sobre la inmortalidad del alma, ${ }^{28}$ aunque desde un punto de vista demostrativo ambos están entrelazados. El último argumento, en clave práctica, tiene una triple forma.

El primer y segundo argumento de Fedón giran en torno al argumento clásico de la inmortalidad del alma en razón a la naturaleza del alma como capacidad racional simple. Concretamente en el primero de los tres argumentos, Mendelssohn muestra como lo simple no puede ser destruido mientras que en el segundo demuestra que el alma es una capacidad simple y no compuesta. ${ }^{29}$

Los tres argumentos del tercer diálogo del Fedón tienen en común una argumentación en términos prácticos. De los tres, únicamente el tercero pertenece originalmente a Mendelssohn. ${ }^{30} \mathrm{El}$ primero de los tres argumentos se basa en la premisa de que el hombre está destinado a obtener una perfección cada vez mayor. Según Altmann esta premisa está directamente tomada del concepto de monada leibniziana que siempre aspira a mayor perfección. ${ }^{31}$ La antropología mendelssohniana parte de un desarrollo positivo imparable desde el mismo naci-

\footnotetext{
${ }^{26}$ Cfr. Feiner, S., Moses Mendelssohn. Ein jüdischer Denker in der Zeit der Aufklärung, trad. Inge Yassur, Göttingen, Vandenhoeck und Ruprecht, 2009, p. 116.

${ }_{27}$ Cfr.. Altmann, A., Moses Mendelssohn. A Biographical Study. London, Routledge and Kegan Paul, 1973, p. 140 y siguientes.

${ }^{28}$ Ibid., p. 144 y p. 154.

${ }^{29}$ Para esta demostración, Mendelssohn se apoya en una demostración de Plotino dotándole de una nueva forma aunque sin alterar su contenido (cfr. Strauss, L., «Einleitung», en JubA 3.1, p. XXIX)

${ }_{30}$ Arkush, A., Mendelssohn and the Enlightenment, Albany, State University of New York Press, 1994, p. 56.

31 Altmann A., Moses Mendelssohn, p. 156.
} 
miento. Los hombres desarrollan nuevas capacidades y educan su capacidad racional, el entendimiento y los sentidos. Esta es la manera de crecer y perfeccionarse. El hombre ha nacido para imitar a Dios y por ello, este progreso individual no puede llegar a un fin en cada individuo por lo que la muerte no ha de suponer una interrupción de este proceso. Entonces, hay que presuponer una continuidad más allá de la muerte corporal, de manera que el hombre pueda seguir formando el potencial que le impulsa al bien. Según Mendelssohn, el hombre está determinado a la perfección y por ende también a la felicidad a través de su acercamiento al ideal divino. Por ello el alma tiene ser inmortal: $:^{32}$

\begin{abstract}
"Mediante la imitación divina nos acercamos paulatinamente a nuestras perfecciones. En este acercamiento consiste precisamente la felicidad de los espíritus. Pero el camino que conduce a ellos es infinito, y no puede ser recorrido en su totalidad ni en toda la eternidad. Por ello el progreso el progreso de la vida humana no conoce límite alguno." 33
\end{abstract}

Mendelssohn asume la existencia de un más allá de manera que el hombre pueda continuar con este proceso de continuo progreso. En este visión, el más allá y este mundo (Jenseits y Diesseits) se hallan mucho más cerca que en las concepciones de la mayoría de autores judíos ya que sin esta cercanía el proceso de perfección no sería posible. Una de las razones de esta posición puede ser el hecho de que en el Judaísmo bíblico y talmúdico se niega una posible ruptura entre lo temporal y lo infinito. ${ }^{34}$ Resumiendo, en su concepto Mendelssohn traslada el pensamiento de la perfectibilidad al ámbito del desarrollo individual, hecho que sale a relucir en la discusión que Mendelssohn mantiene con Thomas Abbt acerca de la determinación de los hombres. ${ }^{35}$

\footnotetext{
${ }^{32}$ Mendelssohn, M., Phaedon oder ueber die Unsterblichkeit der Seele, en JubA 3.1, p.105 y siguientes.

${ }^{33}$ Durch die Nachahmung Gottes kann man sich allmählig seinen Vollkommenheiten nähern, und in dieser Näherung bestehet die Glückseligkeit der Geister; aber der Weg zu denselben ist unendlich, kann in Ewigkeit nicht ganz zurück gelegt werden. Daher kennet das Fortstreben in dem menschlichen Leben keine Grenzen" (ibid., p. 113).

${ }^{34}$ Zac, S., Essence du Judaïsme et liberté de conscience, en, Les nouveaux Cahiers 8/34,1973, pp.14-29, aquí, p. 19.

${ }^{35}$ Para esta demostración, la incitación de Thomas Abbt fue de gran relevancia. En una carta del 22 de julio de 1766 escribe Mendelssohn "Sus preguntas me han animado a escribir un tratado sobre la inmortalidad del alma con cuya elaboración ya comencé hace muchos años. Mis razones las pongo en la boca de Sócrates. Corro el riesgo de hacer de Sokrates un leibniziano. Pero no importa. He de incluir a un pagao para no tener que recurrir a la revelación" (en JubA 12.1, p. 118). En las preguntas de Abbt salen a relucir las dudas de éste sobre la respuesta que Spalding había dado a la cuestión de la determinación del hombre (cfr. Strauss, L., «Einleitung», en Jub 3.1, p. XVI).
} 
El segundo argumento se basa en demostrar que la negación de la inmortalidad del alma implica un cuestionamiento de la providencia divina. En base al primer argumento del tercer diálogo, el recién nombrado argumento de la perfección, si atribuimos por un lado a esta doctrina un carácter de condición necesaria en la determinación de los hombres, negarla, implicaría una contradicción en el concepto de providencia puesto que de acuerdo al concepto de providencia, tal y como será expuesto a continuación, la inmortalidad del alma es necesaria. ${ }^{36}$

En el tercer argumento, el genuino de Mendelssohn, éste recurre al argumento de la filosofía política de la "colisión de deberes". Mendelssohn entiende que se puede incurrir en un conflicto entre las obligaciones éticas y políticas si no se presupone la inmortalidad del alma. Por ejemplo, el conflicto entre la supervivencia y la obligación ética del sacrificio por otro solo puede ser resuelto bajo la suposición de la inmortalidad. Si esta no existiera, el hombre debería de decidirse o bien por su obligación ética y con ello aceptar su propia destrucción o en caso contrario, haciendo omisión de su obligación ética para con los demás, optar por la prosperidad y el perfeccionamiento propios. El conflicto entre estas dos máximas solo puede ser solucionado admitiendo la inmortalidad del alma. ${ }^{37}$

Estos tres últimos argumentos tienen una relevancia especial ya que aquí se plantea una argumentación en términos puramente prácticos. Mendelssohn se apropia de este tipo de pruebas de "convencimiento práctico" (praktische Überführung) tal y como los denomina en la Abhandlung über die Evidenz, por ser más "vivaces" (lebhaft) y poseer mayor "fuerza de convicción" (Beredungskraft). Sin embargo, en la lucha contra la refutación sofista y el materialismo hay que recurrir a argumentos puramente teóricos, caracterizados por su claridad y evidencia. En este sentido, el argumento práctico tiene que ser conducido a una base teórica simple, como son los argumentos de la primera y segunda parte del Fedón.

Por otro lado y de acuerdo con la opinión de Strauss, en el caso de los argumentos teóricos lo importante para Mendelssohn no es señalar a estos argumentos como el non plus ultra de la creencia en la inmortalidad del alma, ${ }^{38}$ sino que según el propio Mendelssohn estos argumentos obedecen más bien a un convencimiento anterior, casi originario y directo: el entendimiento sano.

\footnotetext{
${ }^{36}$ Mendelssohn, M., Phaedon oder ueber die Unsterblichkeit der Seele, en JubA 3.1, p.121.

${ }^{37}$ Mendelssohn, M. Op. cit., JubA 3.1, p.116 y siguientes; cfr. también Altmann, Moses Mendelssohn, p. 156.

${ }^{38}$ Strauss, L., «Einleitung», en JubA 3.1, p. XXV.
} 


\section{Providencia}

El concepto de providencia ha sido esbozado parcialmente al hablar del tercer argumento del Fedón. En el escrito Sache Gottes (La cuestión de Dios) Mendelssohn define el concepto de la providencia. Evidentemente, está en consonancia con sus otros principios antropológicos y metafísicos básicos, aquí ya explicados, así como con sus argumentos. ${ }^{39}$

En líneas generales, la bondad y la justicia de Dios se deducen de los atributos clásicos tales como omnipotencia, independencia y omnisciencia. En consecuencia, Mendelssohn formula una teodicea en la línea de la tradición de Leibniz, si bien difiere del filósofo de la Monadología en algunos aspectos fundamentales. Por ejemplo, Mendelssohn no asume la posibilidad de una condena eterna de los impíos y considera esta doctrina de Leibniz como puramente cristiana. ${ }^{40}$

Si bien Mendelssohn adopta la visión de Leibniz de la justicia como una "bondad guiada por la sabiduría" (Weisheit gelenkte Güte) y comparte su concepto de virtud, se aleja por completo de Leibniz en su visión de la "dureza del castigo" " ${ }^{11}$ algo que Mendelssohn no puede suscribir al rechazar cualquier condena eterna. ${ }^{42}$ Asimismo la idea de un Dios severo y vengativo capaz de permitir un castigo eterno es imposible de acuerdo con su determinación del hombre siempre destinado a la consecución de una mayor felicidad. ${ }^{43}$ En este sentido, Mendelssohn concede que los castigos en el más allá no pueden ser fruto de una sed de venganza de Dios sino de la bondadosa voluntad divina en pro de la mejora de cada individuo, es decir, siempre por el propio beneficio del hombre. Esto puede ser tan solo en cuanto a que el castigo de cada hombre sirve para la continuación de este proceso de perfeccionamiento por tener una función educativa.

Por último, hay que mencionar cómo en este ensayo escrito para su hijo Joseph, pese a las divergencias ya expuestas, Mendelssohn se basa hasta tal punto en el Causa Dei de Leibniz que hay partes que son tomadas directamente, sustituyendo tan sólo las citas de origen cristiano por fuentes judías. ${ }^{44}$ Para Strauss, las diferencias concretas entre los dos autores en lo que respecta a sus conceptos de castigo eterno no son realmente fruto de la diferencia de orígenes

\footnotetext{
${ }^{39}$ Ibid., p. C.

${ }^{40}$ Mendelssohn, M., Sache Gottes, en Jub A 3.2, p. 240.

${ }^{41}$ Mendelssohn, M., Sache Gottes, en JubA 3.2, p. 230 y siguientes.

${ }^{42}$ Ibid., p. 240.

${ }^{43}$ Strauss L. y Bamberger F., «Einleitung», en Jub3.1, p. XIX.

${ }^{44}$ Cfr. Strauss, L., «Einleitung», en JubA 3.2, pp. CI-CII.
} 
religiosos sino que se deben más al hecho que Mendelssohn como "sabio" del siglo XVIII puede distanciarse mucho más la religión positiva que Leibniz en su momento así como al hecho de que el concepto de Dios de Mendelssohn está fuertemente influenciado por el pensamiento ilustrado. ${ }^{45}$

A modo de conclusión, hay que señalar que en La Cuestión de Dios el elemento central de la religión de la razón es la idea de la bondad divina de modo que la felicidad del hombre se considera como el fin último de la creación. ${ }^{46}$

\section{El problema del método metafísico en los asuntos metafísicos}

En la diferenciación hecha por Leibniz entre las vérites de raison y vérités de fait $^{47}$ Mendelssohn reconoce el punto de interconexión entre las matemáticas y la "sabiduría del mundo" (Weltweisheit). Ambas verdades coinciden en su carácter eterno, puesto que lo contrario no puede ser pensado de ellas, es decir no pueden ser negadas. La razón es la única capacidad humana apta para alcanzarlas y comprenderlas. De esto se deriva su carácter tanto necesario como universal, algo válido tanto para las verdades de la metafísica como para las verdades matemáticas. ${ }^{48}$

Junto a estas verdades, existen las verdades contingentes que son siempre obtenidas a través de la observación. Mientras que las verdades necesarias proceden del entendimiento divino, las contingentes lo hacen de la voluntad divina. ${ }^{49}$ Tal y como ya ha sido mencionado, las verdades eternas son universales, necesarias y atemporales por lo que están fijadas y por tanto no sujetas a transformaciones, por lo que la metafísica es una ciencia acabada ${ }^{50}$ una idea por otro lado bastante común entre los pensadores de la Ilustración. ${ }^{51}$

\footnotetext{
${ }^{45}$ Cfr. op. cit., p.CII y Guttmann, J., Die Philosophie des Judentums (1933), Wiesbaden, Fourier 1985, aquí p. 316.

${ }^{46}$ Strauss L., «Einleitung», en JubA 3.2, p. CIII y siguientes. Para Leibniz, el fin de la creación está en la belleza y el orden del todo. (Leibniz, G.W., Essais de Théodicée (1710), en Die philosophischen Schriften (7 tomos), Gebhard C.I. (ed.), Berlin, 1875-1890, reimpresión, Hildesheim/Nueva York, Olms, 1965-1978 (de aquí en adelante GP), aquí VI, párrafo 118 pp. 167-167.).

${ }^{47}$ Leibniz, G.W., Essais de Théodicée (1710), GP VI, párrafos 1-2, pp. 49-50.

${ }_{48}$ Mendelssohn, M., Jerusalem, en JubA 8, pp.158-159.

${ }^{49}$ Mendelssohn, M., Jerusalem JubA 8, p.158 y Sache Gottes JubA 7, p. 224 ; cfr. Altmann, A., Proofs for the Existence of God, p. 16.

${ }^{50} \mathrm{Cfr}$. Cassirer, E., «Die Idee der Religion bei Lessing und Mendelssohn», en Festgabe zum zehnjährigen Bestehen der Akademie für die Wissenschaft des Judentums. 1919-1929. Berlin, Akademie 1929. pp. 22-41, nueva reimpresión en, Gesammelte Werke, Vol.17, pp. 93-114, aquí p. 100.

${ }^{51}$ Cfr. Cassirer, Die Philosophie der Aufklärung, p. 230.
} 
También las verdades matemáticas tienen una naturaleza eterna y necesaria. Sin embargo, las matemáticas tienen un grado de evidencia que las verdades metafísicas no puede obtener. Se ha visto como Mendelssohn constata este hecho al principio de su trayectoria, en la Abhandlung über die Evidenz, que se extiende a lo largo de su obra y concepciones como muestra la evolución y naturaleza de los argumentos de sus principios metafísicos.

Esta deficiencia puede ser considerada el motivo de la argumentación en términos prácticos, tal y como Mendelssohn la desarrolla en la tercera parte de la Abhandlung: una argumentación práctica que no recurre a una prueba tan sólida y convincente sino a una intuición, a la sabiduría popular. Esta valoración de los argumentos "populares" es la novedad que Mendelssohn aporta a su panorama contemporáneo pero es también el hilo y la tendencia de la filosofía mendelssohniana. En este sentido, el argumento de la existencia de Dios basado en la evidencia de tal hecho es, según Strauss, la culminación del dualismo epistemológico subyacente desde la Abhandlung über die Evidenz, ${ }^{52} \mathrm{y}$ que es formulado en el capítulo nueve definitivamente en su última obra.

En este capítulo de las Morgenstunden Mendelssohn explica la relación entre el entendimiento sano y la razón especulativa. La alegoría del sueño (Traumallegorie) de ilustra la disyuntiva que impera entre el sensus communis y la contemplatio. En la discordia entre ambos es la razón la que ha de intervenir y decidir quién está en lo correcto. Algo parecido ocurre entre la especulación y el entendimiento sano. La razón dogmática especulativa puede desviarnos de los "derroteros del sentido común" (Heerstrasse des Gemeinsinns), por ello conviene situarse en un primer momento al lado del entendimiento sano. Sin embargo, este también puede llevar a engaños ya que realiza juicios de manera precipitada e irreflexiva, de ahí la necesidad de recurrir a una capacidad crítica independiente que decide sobre las pretensiones de las capacidades subordinadas, la razón. ${ }^{53}$ El entendimiento sano es un elemento correlativo y correccional a un concepto de razón excesivamente racionalista que recurre en demasía a argumentos especulativos. ${ }^{54}$

Con ello se puede afirmar que la función del entendimiento sano es la de una fuente cognitiva pura así como un saber que procede de manera intuitiva similar a una razón entrenada. ${ }^{55}$ Pero el entendimiento sano tanto como fuente cognitiva

\footnotetext{
${ }^{52}$ Cfr. L.Strauss, «Einleitung», en JubA 3.2, p. XIV. Hütter habla en su tesis doctoral del dualismo como un rasgo constante en la filosofía de Mendelssohn (Hütter, A., Moses Mendelssohn. Philosophie zwischen gemeinen Menschenverstand und unnützer Spekulation, Cuxhaven, Junghans-Verlag, 1990, pp. 41-42).

${ }^{53}$ Mendelssohn, M., Morgenstunden oder Vorlesungen über das Daseyn Gottes, en JubA 3.2, p. 81.

${ }^{54}$ Hütter emplea el término Korrelativ en referencia al entendimiento sano (Hütter, op. cit., p.71).

${ }_{55}^{5}$ Mendelssohn, M, Morgenstunden oder Vorlesungen über das Daseyn Gottes, en JubA 3.2, p.33 y p. 76.
} 
como en su capacidad intuitiva está sujeto al examen de la razón. Su gran ventaja es que a diferencia de una razón que fija sus conocimientos con formas poco ventajosas y no siempre mediante deducciones evidentes, el entendimiento sano es más rápido y asegura así un argumento fácilmente comprensible. ${ }^{56}$ Razón y entendimiento sano son dos facultades abocadas a una comprensión mutua y un examen constante mutuo, limitándose a la vez que se dan la razón.

La evolución de Mendelssohn hasta llegar a una situación de práctica paridad entre razón y entendimiento es para Strauss fruto del empeño de Mendelssohn a justificar el Judaísmo en términos estrictamente racionales. ${ }^{57}$ Contrario a esta tesis, Altmann afirma que Mendelssohn nunca se apartó de la convicción que las verdades de la religión natural pudieran ser alcanzadas por un método demostrativo. Su pensamiento racional no varió, simplemente en él cundió una desilusión cada vez mayor con el more geometrico, puesto que la expresión de teoremas en términos estrictamente teóricos resultaba menos evidente que su formulación como parte del saber práctico del hombre. ${ }^{58}$

Por otro lado, para Strauss existe una analogía entre el conocimiento con orientación práctica y el conocimiento derivado del entendimiento sano.$^{59}$ Esta es una tesis compartida por el presente artículo y que lo acerca además a las posturas de Altmann puesto que estas dos instancias de justificación y demostración finalmente pueden ser concebidas como dos respuestas al mismo problema. En este mismo sentido Edward Breuer opina que el afán constante de Mendelssohn consistió en dar con otra fuente distinta del acceso racional, menos científica, que pudiera salvaguardar la universalidad de los principios de la religión natural a la luz de la relación intrínseca existente entre la felicidad y la religión natural. Por este motivo y partiendo de su convencimiento que la extensión de las verdades de razón es universal y necesaria, es importante para Mendelssohn, según Breuer, poder concebir estas verdades no únicamente en términos racionales con el fin de que tanto los formados en deducción especulativa como los que carecen de estos conocimientos pudieran llegar a comprenderlas. ${ }^{60} \mathrm{Zac}$ denomina esta aspiración como el "rasgo democrático" de la filosofía de Mendelssohn. ${ }^{61}$

\footnotetext{
${ }^{56}$ Rotenstreich, N., Jewish Philosophy in modern times. From Mendelssohn to Rosenzweig, New York/Chicago/San Francisco, Holt, Rinehart and Winston, 1968, aquí p. 19.

${ }^{57}$ L.Strauss, «Einleitung», en JubA 3.2, pp. LXVI y siguientes.

${ }^{58}$ Altmann, A., «Mendelssohn's Concept of Judaism re-examined», en Von der mittelalterlichen zur modernen Aufklärung. Studien zur jüdischen Geistesgeschichte, Tübingen, J.C.B. Mohr, 1987, pp. 234-248, aquí p. 243 y Altmann, Proofs for the Existence of God, p. 29.

${ }^{59}$ Straus, L. «Einleitung», en Jub 3.2, LXVI.

${ }^{60}$ Breuer, E., Rabbinic Law and Spirituality. Jewish Quarterly Review 56, 1996, 3, pp. 299321, aquí p. 299.

${ }^{61}$ Zac, S., op. cit., p. 26.
} 
La enfatización del entendimiento sano se nutre además de la experiencia de la insuficiencia del lenguaje de la demostración dogmática y el peligro derivado de ello en forma de "entusiasmo" (Schwärmerei) ${ }^{62}$ En relación a esto Beiser reconoce un malestar debido a la creciente desunión entre la creencia popular y la filosofía: ${ }^{63}$

"Cuando hablo de una convicción acorde con la razón y presupongo que estas no son puestas en duda en el Judaísmo, no hablo de una argumentación metafísica tal y como estamos acostumbrados a encontrarnos en libros; tampoco hablo de demostraciones acordes con nuestra escuela que han pasado por todas las pruebas del más sutil de los espíritus dubitativos, sino que hablo de las frases y los juicios de un mero entendimiento sano, que los toma en consideración y reflexiona sobre ellos tranquilamente. Es cierto que soy un gran venerador de las demostraciones metafísicas y estoy firmemente convencido de que las principales verdades de la religión natural pueden ser probadas de manera igual mente apodíctica que cualquier otro teorema de la teoría de las dimensiones. Sin embargo, mi convección sobre las verdades religiosas no depende hasta tal forma de una argumentación metafísica de modo que se le vaya todo en ella " "64

Consciente de la impenetrabilidad de algunas argumentaciones especulativas y de la relación vital entre la religión y la felicidad, la filosofía mendelssohniana es lo que se podría denominar una filosofía consoladora. El importante rol del entendimiento sano tiene la tarea de asegurar por igual un acceso a la felicidad, ${ }^{65}$ de forma que el conocimiento quede atado al designio

\footnotetext{
${ }^{62}$ Mendelssohn, M., An die Freunde Lessings, en Jub3.2, p. 197 así como la carta a Johann Georg Zimmerman (Brief an Johann Georg Zimmerman 1. September 1784, en JubA 13, pp. 221-222.

${ }^{63}$ Beiser, F. C., The Fate of Reason. Cambridge, Harvard University Press 1987, p. 100.

64 "Wenn ich aber von vernunftmäßiger Ueberzeugung rede, und solche im Judenthum als unbezweifelt voraus setzen will; so ist die Rede nicht von metaphysischer Argumentation, wie wir sie in Büchern zu führen gewohnt sind; nicht von schulgerechten Demonstrationen, die alle Proben des subtilsten Zweifelmuths bestanden sind; sondern von den Aussprüchen und Urtheilen eines schlichten gesunden Menschenverstandes, der die Dinge ins Auge faßt und ruhig überlegt. Zwar bin ich ein großer Verehrer der Demonstrationen in der Metaphysik, und fest überzeugt, daß die Hauptwahrheiten der natürlichen Religion so apodiktisch erweislich sind, als irgend ein Satz in der Größenlehre. Gleichwohl aber hängt selbst meine Ueberzeugung von Religionswahrheiten nicht so schlechterdings von metaphysischen Argumentationen ab, daß sie mit denselben stehen und fallen müßte" (Mendelssohn, M., An die Freunde Lessings, en JubA 3.2, p. 197)

${ }^{65}$ Cfr. Hütter, op. cit., S.43.
} 
del hombre. ${ }^{66}$ En esta constelación, el racionalismo solo puede aspirar a ser un medio para un fin. ${ }^{67}$

En el plano histórico se puede observar como un motivo más para la posición ciertamente escéptica de Mendelssohn hacia el pensamiento racional se halla en la misma crisis de la metafísica. En este mismo sentido, Allan Arkush señala como la respuesta mendelssohniana es por un lado una réplica del dogmatismo irracional de Jacobi basado únicamente en la fe pero es también una respuesta al desafío kantiano. A través de una enfatización del entendimiento sano, Mendelsoshn intenta contraatacar la supuesta insuficiencia de la razón postulada por Kant, con el mero fin de asegurar un estatus cognitivo a las verdades metafísicas, ${ }^{68}$ siempre al servicio de la felicidad humana ${ }^{69}$

\section{El concepto de revelación}

Dios, alma y providencia son los principios metafísicos de la religión de la razón a cuya demostración Mendelssohn dedicó gran parte de sus esfuerzos. Junto a estos conceptos hay otro pilar fundamental en el edificio teórico de Mendelssohn perteneciente al ámbito estrictamente religioso o de la filosofía de la religión. Se trata del concepto de revelación.

En su obra Jerusalem Mendelssohn determina el espacio concreto de la revelación. ${ }^{70}$ Para ello son dos los aspectos relevantes. Por un lado, no cabe la posibilidad de que haya habido una revelación exclusiva de verdades eternas. Tal y como señala el filósofo de Dessau, las verdades de razón tienen que poder ser comprendidas y alcanzadas por todos. La demostración especulativa o simple evidencia en forma de conocimiento orientado a la práctica o entendimiento sano aseguran esta comprensión. Desde un punto de vista ético la existencia de tal revelación también se torna imposible. ${ }^{71} \mathrm{Si}$ de acuerdo a la

\footnotetext{
${ }^{66}$ Cfr. Hütter, op. cit., p.20 und Böhr, C., «Johann Jakob Engel und die Geschichtsphilosophie Moses Mendelssohns» en Albrecht M., Engel, E.J., Hinske, N. (Eds.), Moses Mendelssohn und die Kreise seiner Wirksamkeit. Tübingen, De Gruyter, 1994, pp. 157-174, aquí p. 171.

${ }^{67}$ Schweid, E., History of Modern Jewish religious Philosophy, Vol.1: The Period of Enlightenment, trad. Leonard Levin, Leiden/Boston, Brill, 2001, p. 103.

${ }^{68}$ Arkush, A., op. cit., p. 75 y p. 82.

${ }^{69}$ Cfr. Carta a Zimmerman del 1 de septiembre de 1784 (Brief an Zimmerman vom 1. September 1784, en Jub13, pp. 221-222. Cfr. Strauss, L. «Einleitung», en Jub 3.2, LXIX und Altmann, A., Proofs for the Existence of God, p. 29.

${ }^{70}$ Cfr. Altmann, «Einleitung», en Jub 8, p. XLII.

${ }^{71}$ Cfr. Guttmann, J., Mendelssohns Jerusalem und Spinozas theologisch-politischer Traktat, en 48. Bericht der Hochschule für Wissenschaft des Judentums in Berlin, 1931, pp. 31-67, aquí p. 43.
} 
definición de Mendelssohn la providencia es justa y bondadosa, ${ }^{72}$ resulta entonces incompatible con su propio concepto que Dios pueda revelar verdades necesarias de manera exclusiva a un solo grupo al tratarse de verdades necesarias para la salvación y la felicidad. Si realmente así fuera, no solo la beatitud del más allá sino la de este mundo dejarían de ser posibles, al menos para la mayor parte de los humanos. ${ }^{73}$

Como Mendelssohn señala en Jerusalem, el concepto de Dios, la inmortalidad del alma y la providencia son indispensables para la felicidad humana y su conocimiento no puede por tanto depender de una revelación particular. ${ }^{74}$ Dios tiene que haber dotado a todos los hombres de la "divina razón" y de los medios para alcanzar la felicidad, una felicidad basada en los principios de la razón y la religión natural:

"No creo que las fuerzas de la razón humana sean insuficientes para convencerla de las verdades eternas imprescindibles para la felicidad humana y que por tanto Dios haya tenido que revelárselas de una manera sobre natural.’’75

Las verdades necesarias para la felicidad humana solo pueden y deben ser alcanzadas por un medio puramente humano y tan solo la razón tiene esta extensión universal al no estar, al contrario que una revelación particular, restringida a un cierto número de hombres, es decir, conforme a la última cita, al ser una revelación "natural" y no "sobrenatural".

De acuerdo a esta premisa ética, una revelación exclusiva de verdades eternas es simplemente imposible. Sin embargo, nada contradice una revelación particular y sobrenatural de verdades que la razón pueda alcanzar por sí misma, en lo que sería algo así como una repetición o confirmación de las mismas. No obstante, Mendelssohn también quiere destruir la posibilidad de una revelación de verdades necesarias pero no exclusiva, tal y como fue concebida por lo deístas y neólogos. ${ }^{76}$ No hay punto de unión posible entre la revelación sobrenatural y la verdad necesaria, ni repetición posible de verdades necesarias

\footnotetext{
${ }^{72}$ Cfr. Guttmann, J., Philosophie des Judentums, p. 327.

${ }^{73}$ Mendelssohn, M., Jerusalem, en JubA 8, S.160f., p. 201.

${ }^{74}$ Ibid., p. 131.

75 "Ich glaube also nicht, daß die Kräfte der menschlichen Vernunft nicht hinreichen, sie von den ewigen Wahrheiten zu überführen, die zur menschlichen Glückseligkeit unentbehrlich sind, und daß Gott ihnen solche auf eine übernatürliche Weise habe offenbaren müssen“" (Ibid., pp.160-161).

${ }^{76}$ Los Neólogos o la Neología es un movimiento de teólogos protestantes alemanes del siglo XVIII que intentaron acercarse a las ideas de la ilustración a través de una reformulación de los dogmas cristianos o incluso a menudo una relativización. Una buena monografía sobre el
} 
en una revelación. ${ }^{77}$ De este modo Mendelssohn se aleja definitivamente de la posibilidad de tomar una posición intermedia entre la "Teología de la revelación" y el Deísmo. ${ }^{78} \mathrm{La}$ imposible unión se debe en primer lugar a una cuestión epistemológica. Mendelssohn toma de Leibniz las categorías de verdades necesarias y contingentes a las que añade la categoría de verdad histórica. ${ }^{79}$ Las verdades históricas están dadas empíricamente, pero al contrario que las contingentes, no pueden ser obtenidas por observación constante al hallarse en el pasado. Por este motivo, es imposible que tengan un carácter necesario. Son dadas una vez y desaparecen para siempre, estando sometidas a una condicionalidad temporal y espacial. ${ }^{80}$ La revelación es necesariamente una verdad histórica, porque se halla en el pasado y la única manera de acceder al conocimiento de la misma es a través de la tradición y la transmisión histórica. No obstante, en un hecho histórico no pueden ser depositadas verdades eternas, por lo que el contenido epistemológico ha de ser distinto del de una verdad de razón. ${ }^{81}$ Las verdades históricas del pasado no pueden ser por tanto alcanzadas a través de la razón o la observación del presente. Aún así, pueden ser actualizadas mediante la transmisión de testimonios fiables. Para ello, el testigo ha de cumplir una serie de condiciones que Mendelssohn enumera en las Gegenbetrachtungen de modo que la fiabilidad del documento pueda ser corroborada, tales como que el que conoce el hecho tuvo que estar presente, tiene que tener sentidos sanos, no estar dominado por afectos y haber examinado el hecho atestiguado. ${ }^{82}$ Sólo así puede ser establecida la autoridad de un docu-

pensamiento religioso alemán ilustrado del siglo XVIII se encuentra en la obra Feiereis, K., Die Umprägung der natürlichen Theologie in Religionsphilosophie; ein Beitrag zur deutschen Geistesgeschichte des 18. Jahrhunderts, Leipzig, St-Benno Verlag, 1965.

${ }^{77}$ Cfr. Bourel, op. cit., p. 338, Guttman, J., Philosophie des Judentums, p. 321 y Altmann, A., «Einleitung», en JubA 8, p. XLVI.

${ }^{78}$ Altmann, A., «Einleitung», Jub A8, p. XLVI.

${ }^{79}$ Cfr. Hilfrich, C., Lebendige Schrift. Repräsentation und Idolatrie in Moses Mendelssohns Philosophie und Exegese des Judentums, München, Wilhelm Fink Verlag, 2000, p. 79. En Morgenstunden Mendelsohn hace la diferenciación entre los dos tipos de verdades. Las leyes de la naturaleza son contigentes y eternas mientras que las históricas son contingentes y temporales (Mendelssohn, M., Morgenstunden oder Vorlesungen über das Daseyn Gottes, en JubA 3.2, p. 75).

${ }^{80}$ Mendelsohn, M., Jerusalem oder über religiöse Macht und Judentum, en JubA 8, pp.159160 y Morgenstunden oder Vorlesungen über das Daseyn Gottes, en JubA 3.2, p. 90; cfr. Breuer, E., Of Miracles and Events Past: Mendelssohn on History, en Jewish History, 9, 1995, pp. 27-52, aquí pp. 42-43.

${ }_{81}$ Mendelssohn, M., Jerusalem oder über religiöse Macht und Judentum, en JubA 8, pp.159160, Cfr. Breuer, E., Mendelssohn on History, p. 32.

82 "Los hombres pueden conocer sucesos cuando 1) están presentes, 2) tienen sanos los sentidos 3 ) no tiene afectos, 4) y han investigado el asunto" ("Die Menschen können sich von Begebenheiten unterrichten, 1) wen sie gegenwärtig sind, 2) gesunde Sine haben, 3) von Affekten frey sind, 4) und die Sache untersucht haben“), en Mendelssohn, M., Gegenbetrachtungen über Bonnets Palingenesie, en JubA 7, p. 84. 
mento. Una revelación y los milagros que la acompañan son únicamente validos como verdad histórica si estas condiciones se cumplen. Si la validez de los hechos ha sido confirmada, lo que no siempre resulta una tarea fácil a la vista de posibles contiendas sobre la validez de los testimonios, este hecho solo puede, sin embargo, reclamar para sí el carácter de verdad histórica ${ }^{83}$

En la definición de milagro de Mendelssohn ${ }^{84}$ aflora de nuevo la imposibilidad de que una verdad histórica aunque haya sido examinada por este proceso, pueda afirmar algo de una verdad necesaria o incluso llegar a probarla. En esta definición, Mendelssohn afirma que los milagros solo pueden probar intenciones pero no doctrinas ${ }^{85}$ Una revelación no puede entonces añadir o probar nada de las verdades de razón y por ende no puede tener una pretensión universal, ya que la religión humana universal no debería ser sometida a la limitación de una religión revelada. El criterio de su verdad nunca será una confirmación externa. ${ }^{86}$

El trasfondo de estas reflexiones de Mendelssohn no es otro que la incompatibilidad de dos ámbitos, el de las verdades históricas y el de las verdades necesarias. Como ha sido mencionado, una verdad histórica está sujeta a un tiempo y un espacio, algo imposible para una verdad necesaria de la razón. La aportación de Mendelssohn al negar una coincidencia entre ambos planos, el histórico y el necesario, refleja la imposibilidad epistemológica de mezclar ambos tipos de verdades a lo que se suma su diferente carácter ontológico, de manera que no pueden ser alcanzados en un mismo plano epistemológico:

"Siempre que las intenciones divinas sean que los hombres sean convencidos de una verdad, la sabiduría divina dotará a los hombres de los medios para alcanzarla. Si se trata de una verdad necesaria, les dotará del grado necesario de razón. Si es una ley natural lo que tienen que conocer, entonces les dotará con el espíritu de la observación y si se trata de una

${ }^{83}$ Mendelssohn, M., Gegenbetrachtungen über Bonnets Palingenesie, en JubA 7, pp.84-85. 84 “QQue pueden demostrar los milagros? - Pueden dar a conocer la voluntad de un ser sobrehumano; pues prueban la decisión y e intenciones de un ser sobrehumano, pero no pueden confirmar doctrinas. Ninguna intervención de Dios en el rumbo de la naturaleza ha sido un milagro; puesto que no tenía que ninguna intención especial ("Was können Wunder beweisen? - Sie können den Willen eines übermenschlichen Wesens zu erkennen geben; denn sie beweisen Vorsatz und Absichten eines übermenschichen Wesens, bestätigen aber keine Lehren. Jede Einwirkung Gottes in den Lauf der Natur war kein Wunder; in so weit sie keine besondere Absicht zu erkennen geben würde“"), en Mendelssohn, M., Ueber Wunder u[nd] wunderbar, en JubA 6.1, pp. 4-5)

${ }^{85}$ Mendelssohn, M., Ueber Wunder u[nd] wunderbar, en JubA 6.1, pp. 4-5.

${ }^{86}$ Vetter, D., «Religion als Ethik? Zur Problematik von Moses Mendelssohns Bestimmung des Judentums», en Müller, W.E., y Schulz, H. (eds,), Theologie und Aufklärung, Würzburg, Königshaysen \& Neumann, 1992, pp. 89-111, aquí p. 94. 
verdad histórica que ha de ser conservada para la posteridad, confirmará su certeza histórica fijando la credibilidad del narrador por encima de toda duda." ${ }^{\prime 87}$

También Lessing formuló en varias ocasiones razonamientos similares. Entre otros acuñó la famosa frase "las verdades históricas contingentes nunca pueden llegar a ser la prueba de verdades de razón"\$8. Volviendo a Mendelssohn, aparte de la incompatibilidad epistemológica general, las verdades históricas no son aptas para verdades de razón por otros motivos más prácticos. En primer lugar, puede darse una contienda entre testimonios. Entonces, se pregunta Mendelssohn ¿como los testimonios pueden contener información necesaria, si los testimonios se contradicen a menudo? ¿Cómo puede la felicidad de cada cual depender de un testimonio, tan pronto confirmado como invalidado? Las verdades de la razón son claras y evidentes y no admiten que se piense lo opuesto a ellas. Los testimonios, más allá de que puedan entumecer a los mismos conceptos, pueden ser falseados, ${ }^{89}$ por lo que es imposible que hagan justicia a las verdades necesarias de la razón.

En segundo lugar, Mendelssohn realiza un giro a la argumentación clásica cuando afirma que no son los milagros los que dan fe de las verdades, sino que el acontecimiento de la revelación y los milagros ahí acontecidos pueden solo ser percibidos si previamente se han asumido las verdades necesarias, a saber, que hay un Dios que se revela. ${ }^{90}$

Finalmente hay que señalar de nuevo como para Mendelssohn no hay religión alguna que debido a la recepción de una revelación concreta pueda afirmar disponer de ciertas verdades que no estén disponibles para resto. La exigencia moral de poner a disposición de todos los hombres las mismas verdades es la premisa básica de las argumentaciones mendelssohnianas. No obs-

\footnotetext{
87 "So oft es nun den Absichten Gottes gemäß ist, daß die Menschen von irgend einer Wahrheit überführt sein sollen; so verleiht ihnen seine Weisheit auch die schicklichsten mittel zu derselben zu gelangen. Ist es eine notwendige Wahrheit, so verleiht sie den dazu erforderlichen Grad der Vernunft. Soll ihnen ein Naturgesetz bekannt werden, so gibt sie ihnen den Geist der Beobachtung; und soll eine Geschichtswahrheit der Nachwelt aufbehalten werden, so bestätigt sie ihre historische Gewißheit, und setzt die Glaubwürdigkeit der Erzähler über alle Zweifel hinweg." (Mendelssohn, M, Jerusalem, en JubA 8, p.160).

${ }^{88}$ Lessing, G.E., Ueber den Beweis des Geistes und der Kraft an den Herrn Director Schumann, zu Hannover. en, Lachmann, K. y Muncker, F. (eds.), Sämtliche Schriften, Stuttgart/ Berlin/ Leipzig, Göschen 1886-1924, reimpresión Berlin, De Gruyter, 1968. Aquí vol. XIII, p. 5.

${ }^{89}$ Mendelssohn, M., Gegenbetrachtungen über Bonnets Palingenesie, en JubA 7, p. 85.

${ }^{90}$ Mendelssohn, M., Gegenbetrachtungen über Bonnets Palingenesie, en JubA 7, S.85. Jospe, E., Moses Mendelssohn: Some reflections on his thought, en Judaism 30, 1981, 2. pp. 168-182, aquí p. 177.
} 
tante, la denominación de revelación como hecho y verdad puramente históricos no tiene por qué significar una degradación de las mismas, tal y como Mendelssohn muestra en Jerusalem. La revelación sinaítica jugará aquí un papel central en la configuración del judaísmo como designio particular de un pueblo.

\title{
6. Conclusión
}

La metafísica de Mendelssohn se mueve entre dos frentes. Por un lado, la inmortalidad del alma, Dios y la providencia conforman los principios de la religión de la razón cuya demostración racional ocupó gran parte de los esfuerzos de la filosofía ilustrada alemana. Por otro, Mendelssohn desarrolla junto a las demostraciones clásicas otro tipo de demostraciones en clave práctica o fundadas en el entendimiento sano como fuente de conocimiento. La intención suya dista mucho de ser la del pensador dogmático de la contra-ilustración que pretendía asegurar las verdades per se, por su mero hecho teórico, a modo del "entusiasta", sino que según la aspiración última del ilustrado de Dessau hay que evitar estos tres principios sean derribados por ser los pilares de la felicidad humana. Esta es la razón por la que también rechaza una revelación de verdades metafísicas, comunes a todos los hombres de todos los tiempos y algo que acarreará consecuencias de gran alcance en su concepto del Judaísmo. Pero si estas tres verdades han de estar a disposición de todos los hombres de todos los tiempos, no pueden depender únicamente de una demostración genuinamente filosófica propia de los círculos académicos, sino que hay que entregarlas a una fuente de conocimiento más intuitiva y por ende más simple como es el entendimiento sano. El pensamiento mendelssohniano apunta a una primacía de la argumentación en clave práctica con respecto a la teórica. Pero quizás más importante es aún la constatación que tanto para el pensamiento religioso como metafísico el único fin en sí a tener en cuenta es la felicidad. Este es la novedad y particularidad mendelssohniana y razón por la que en palabras de Altmann, su ilustración es la ilustración consoladora.

\author{
Pablo Alejandro Arias PÉREZ \\ Departamento de Historia de la Filosofía \\ Universidad Complutense de Madrid \\ a_plein_soleil@hotmail.com
}

